TRANSACTIONS OF THE

AMERICAN MATHEMATICAL SOCIETY

Volume 356, Number 5, Pages 1889-1907

S 0002-9947(03)03234-3

Article electronically published on October 6, 2003

\title{
ASYMPTOTIC BEHAVIOR OF THE SOLUTIONS OF LINEAR AND QUASILINEAR ELLIPTIC EQUATIONS ON $\mathbb{R}^{N}$
}

\author{
PATRICK J. RABIER
}

\begin{abstract}
We investigate the relationship between the decay at infinity of the right-hand side $f$ and solutions $u$ of an equation $L u=f$ when $L$ is a second order elliptic operator on $\mathbb{R}^{N}$. It is shown that when $L$ is Fredholm, $u$ inherits the type of decay of $f$ (for instance, exponential, or power-like). In particular, the generalized eigenfunctions associated with all the Fredholm eigenvalues of $L$, isolated or not, decay exponentially. No use is made of spectral theory. The result is next extended when $L$ is replaced by a Fredholm quasilinear operator. Various generalizations to other unbounded domains, higher order operators or elliptic systems are possible and briefly alluded to, but not discussed in detail.
\end{abstract}

\section{INTRODUCTION}

We investigate the relationship between the decay at infinity of the right-hand side $f$ and solutions $u$ of an equation $L u=f$ when

$$
L=-\sum_{i, j=1}^{N} a_{i j}(x) \partial_{i j}^{2}+\sum_{i=1}^{N} b_{i}(x) \partial_{i}+c(x)
$$

is a second order linear elliptic operator on $\mathbb{R}^{N}$. Here, $L$ is viewed as a bounded operator from $W^{2, p}$ to $L^{p}$ with $p \in(1, \infty)$, and $L^{p}, W^{2, p}$, etc., denote $L^{p}\left(\mathbb{R}^{N}\right)$, $W^{2, p}\left(\mathbb{R}^{N}\right)$, etc. All those spaces consist of complex valued functions.

We do not follow the traditional treatments of related phenomena, where the asymptotic behavior of (generalized) eigenfunctions is usually derived from properties of the resolvent, for instance in the form of estimates for its kernel. See Glazman 18], Simon [32, or the recent survey of Hislop [20] for Schrödinger operators and Angenent [4] for general elliptic systems.

Neither spectral theory nor semigroups are involved in our arguments, which, conceptually at least, are very simple. Nevertheless, the results are not only more general, but also sharper than most results in the current literature. Of course, the latter statement does not apply to all of the specialized work on Schrödinger operators, which contains various refinements not covered by our analysis.

Recall that an operator $T \in \mathcal{L}(X, Y)$ with $X$ and $Y$ Banach spaces is said to be Fredholm if both $\operatorname{dim} \operatorname{ker} T$ and codim $\operatorname{rge} T$ are finite (so that $\operatorname{rge} T$ is closed). The

Received by the editors September 4, 2001 and, in revised form, August 24, 2002.

2000 Mathematics Subject Classification. Primary 35P05, 35Q40, 47F05.

Key words and phrases. Fredholm operator, unique continuation, eigenvalue, generalized eigenfunction, exponential decay. 
index $\nu$ of $T$ is then defined by

$$
\nu:=\operatorname{dim} \operatorname{ker} T-\operatorname{codim} \operatorname{rge} T \in \mathbb{Z} .
$$

As is well-known (Kato 22], Lindenstrauss and Tzafriri [24]), the set of Fredholm operators of index $\nu \in \mathbb{Z}$ is open in $\mathcal{L}(X, Y)$ (local constancy of the index). When $T=L \in \mathcal{L}\left(W^{2, p}, L^{p}\right)$ and $L$ is also viewed as an unbounded operator on $L^{p}$ with domain $W^{2, p}$, a Fredholm eigenvalue of $L$ is henceforth defined as an eigenvalue $\lambda$ of $L$ such that $L-\lambda$ is Fredholm 1 and the index of $\lambda$ as the index of $L-\lambda$.

The decay of the right-hand side will be accounted for by the condition $e^{t \rho} f \in L^{p}$, where $t>0$ is a real parameter and $\rho \geq 0$ is a chosen $C^{2}$ function $\left(\rho \in W_{l o c}^{2, \infty}\right.$ actually suffices) with first and second derivatives uniformly bounded on $\mathbb{R}^{N}$. For instance, if $\rho(x)=|x|$ for $|x|$ large enough, then $e^{t \rho} f \in L^{p}$ means that $e^{t|x|} f \in L^{p}$ (exponential decay). The choice $\rho(x)=\log |x|$ for $|x|$ large enough corresponds to $|x|^{t} f \in L^{p}$ (power-like decay).

Our approach relies on two main ingredients. The first one is the use of the perturbed operators

$$
L_{s} v:=e^{s \rho} L\left(e^{-s \rho} v\right)
$$

where $s>0$ is a small real parameter, to prove the desired asymptotic behavior when $L$ is either surjective or injective. An operator $L_{\psi} v:=e^{\psi} L\left(e^{-\psi} v\right)$ reminiscent of $L_{s}$ above is used in Agmon's method for proving the exponential decay of eigenfunctions associated with a (real) eigenvalue lying below the essential spectrum of a Schrödinger operator 2 when $p=2$ (see Agmon [1], or Hislop and Sigal [21]), but our use of $L_{s}$ is very different here.

The second ingredient is an approximation theorem showing the existence of a function $d \in C_{0}^{\infty}$ such that $L+d$ is surjective (resp. injective) if index $L \geq 0$ (resp. index $L \leq 0)$, which reduces the problem to the case resolved earlier.

In spite of their generality, the decay properties obtained by the method outlined above are rather precise. Specifically, we show that if $e^{t \rho} f \in L^{p}$ for some $t>0$, then the solutions $u \in W^{2, p}$ of $L u=f$ have the form $u=e^{-s \rho} v$ for some $v \in W^{2, p}$ and $s=\min \left(s_{0}, t\right)$, where $s_{0}>0$ is independent of $f$ and $t$. For instance, pointwise decay of $u$ follows if $\lim _{|x| \rightarrow \infty} \rho(x)=\infty$ and $p>N / 2$ (and also of $\nabla u$ if $p>N$ ). To our best knowledge, the literature covers only the exponential decay of (generalized) eigenfunctions (in particular, $f=0$ ) at isolated eigenvalues of finite multiplicity, and yields $u=e^{-s|x|} v$ with $v \in L^{p}$ or $v \in L^{\infty}$, but not $v \in W^{2, p}$, even when $L$ is a Schrödinger operator. More generally, our result shows for instance that the type of decay of $f$ in a given direction is preserved in the solutions of $L u=f$.

If $L$ is closed (as an unbounded operator on $L^{p}$ with domain $W^{2, p}$ ) and $\lambda$ is an isolated eigenvalue of finite multiplicity, it follows from [22, Theorem 5.28, p. 239] that $L-\lambda$ is Fredholm, and even Fredholm of index 0 by the local constancy of the index. Thus, isolated eigenvalues of finite multiplicity are Fredholm eigenvalues of index 0. The converse is not true: It may happen that $L-\lambda$ is Fredholm of index 0 , yet $\lambda$ is not an isolated point of $\sigma(L)$. In that case, $\sigma(L)$ contains an entire neighborhood of $\lambda$ in $\mathbb{C}$ ([22, Theorem 5.31, p. 241]). Evidently, this cannot occur when $\sigma(L)$ has empty interior (e.g., $p=2$ and $L$ is selfadjoint). In that case,

\footnotetext{
${ }^{1}$ It is easily seen that if $L-\lambda$ is Fredholm for some $\lambda \in \mathbb{C}$, then $L-\lambda$, and hence also $L$, is closed.

${ }^{2}$ Hence isolated and of finite multiplicity.
} 
isolated eigenvalues of finite multiplicity and Fredholm eigenvalues of index 0 are the same.

On the other hand, if $\lambda$ is a Fredholm eigenvalue of $L$ of index $\nu \neq 0$, then $L-\mu$ remains a Fredholm operator of index $\nu$ for $\mu$ in an open neighborhood $U_{\lambda}$ of $\lambda$ in $\mathbb{C}$ and hence the spectrum of $L$ contains $U_{\lambda}$. Therefore, Fredholm eigenvalues of $L$ of nonzero index are never isolated in $\sigma(L)$ (and do not exist when $\sigma(L)$ has empty interior).

In the next section, we consider the case when $L$ is Fredholm and either surjective or injective. The basic approximation theorem mentioned earlier is proved in Section 3 (Theorem 3.3), and the general decay theorem (Theorem 3.4 follows as a corollary. The exponential decay of generalized eigenfunctions associated with arbitrary Fredholm eigenvalues is discussed in Section 4

Some complements are collected in Section 5, that address the $p$-independence of our results or their possible generalizations. We also give simple examples with nonzero index and one showing that ellipticity alone does not guarantee exponential decay, even when the coefficients are constant.

The primary motivation for studying linear elliptic operators via their Fredholm properties is due to the importance of that concept in nonlinear problems. Based on the linear theory, we show in Section 6 that if $p \in(N, \infty)$, the asymptotic behavior is the same as in the linear case for the solutions $u$ of $F(u)=f$ when the quasilinear operator $F$ is Fredholm and $F(0)=0$. This is obtained by rewriting $F(u)=L(u) u$, where $L(u) \in \mathcal{L}\left(W^{2, p}, L^{p}\right)$.

A key feature is that, in a broad setting, $F$ and $L(u)$ are Fredholm if and only if $L(0)=D F(0)$ is Fredholm. Accordingly, the fact that $u$ is unknown is immaterial to $L(u)$ being Fredholm. In contrast, not only the condition that $L(u)$ either is invertible or has 0 as an isolated eigenvalue of finite multiplicity is much more restrictive (it covers only part of the index 0 case), but no verifiable criterion exists that ensures its validity 3 without knowing $u$. Therefore, the decay results in the linear Fredholm case, not merely at isolated eigenvalues of finite multiplicity, are needed to obtain a useful generalization to quasilinear problems, even when the index is 0 .

The last section is mostly an appendix. It is devoted to an elementary proof (based on the operators $L_{s}$ above) that eigenfunctions associated with eigenvalues of finite multiplicity have exponential decay. This is done under assumptions weaker than those needed in Section 3 in some important respects. No use is made of the approximation Theorem 3.3, so that the same elementary argument can be used with general elliptic systems of arbitrary order. This sharpens and generalizes a recent result by Angenent [4].

\section{Decay of SOLUTions When $L$ IS SURJECTIVE OR INJECTIVE}

The explicit assumptions about the coefficients of $L$ needed in this section are rather weak, although further limitation will implicitly arise from the hypothesis that $L$ is Fredholm. We assume only that

$$
a_{i j}, b_{i}, c \in L^{\infty}
$$

\footnotetext{
${ }^{3}$ Except of course when it is equivalent to $L(u)$ being Fredholm of index 0, that is, when the spectrum of $L(u)$ is known to have empty interior.
} 
all complex-valued. This suffices to ensure that, given $p \in(1, \infty), L$ is well defined in $\mathcal{L}\left(W^{2, p}, L^{p}\right)$. In fact, it suffices that $b_{i} \in L^{q_{i}}$ and $c \in L^{q}$ with $\max (N, p)<q_{i} \leq \infty$ and $\max (N / 2, p)<q \leq \infty$ or, more generally, that $b_{i}$ and $c$ are in (finite) sums of spaces $L^{q_{i}}$ and $L^{q}$ as above, respectively. However, we confine our discussion to the case when (2.1) holds, since these assumptions are independent of $p$. For the results of this section, there is no need to formulate any ellipticity condition for $L$.

The $C^{2}$ function $\rho \geq 0$ satisfying

$$
D^{\kappa} \rho \in L^{\infty} \text { for }|\kappa|=1,2
$$

is chosen once and for all. It is straightforward to check that $L_{s}$ in (1.2) is a differential operator with the same properties as $L$ irrespective of $s \in \mathbb{R}$. In fact, the coefficients of $L_{s}$ do not depend upon the function $e^{s \rho}$, and the second order coefficients of $L$ and $L_{s}$ are the same (see (2.3) below). Thus, $L_{s} \in \mathcal{L}\left(W^{2, p}, L^{p}\right)$.

Lemma 2.1. For every $p \in(1, \infty), \lim _{s \rightarrow 0} L_{s}=L$ in $\mathcal{L}\left(W^{2, p}, L^{p}\right)$.

Proof. It is convenient to rewrite $L u$ in the form

$$
L u=-\operatorname{Tr}\left(A D^{2} u\right)+b \cdot \nabla u+c u,
$$

where $A(x):=\left(a_{i j}(x)\right)$ and $b(x):=\left(b_{i}(x)\right)$. Given any $C^{2}$ function $\psi$, the operator $L_{\psi}$ defined by $L_{\psi} u:=e^{\psi} L\left(e^{-\psi} u\right)$ has the explicit expression

$$
\begin{aligned}
L_{\psi} u=L u+\operatorname{Tr}(A(D \psi \otimes D u+D u \otimes D \psi)) & \\
+ & \left(\operatorname{Tr}\left(A\left(D^{2} \psi-D \psi \otimes D \psi\right)\right)-(b \cdot \nabla \psi)\right) u .
\end{aligned}
$$

In particular, if $\psi=s \rho$, then $L_{\psi}=L_{s}$ and

$$
\begin{aligned}
L_{s} u=L u+s \operatorname{Tr}(A(D \rho \otimes D u & +D u \otimes D \rho)) \\
& +s\left(\operatorname{Tr}\left(A\left(D^{2} \rho-s D \rho \otimes D \rho\right)\right)-(b \cdot \nabla \rho)\right) u .
\end{aligned}
$$

Since $A, D \rho$ and $D^{2} \rho$ are uniformly bounded on $\mathbb{R}^{N}$, this shows that

$$
\left|\left(L_{s}-L\right) u\right|_{0, p} \leq\left. C|s||| u\right|_{1, p} \leq\left. C|s||| u\right|_{2, p},
$$

where $C>0$ is a constant independent of $u \in W^{2, p}$ and of $s$ with (say) $|s| \leq 1$. It follows that $\left\|L_{s}-L\right\|_{\mathcal{L}\left(W^{2, p}, L^{p}\right)} \leq C|s|$ for $|s| \leq 1$. This completes the proof.

We begin with the case when $L$ is surjective.

Theorem 2.2. Let $p \in(1, \infty)$ be given. Suppose that $L \in \mathcal{L}\left(W^{2, p}, L^{p}\right)$ is Fredholm and surjective (hence of index $\nu \geq 0$ ). Then, there is $s_{0}>0$ such that whenever $f \in L^{p}$ satisfies $e^{t \rho} f \in L^{p}$ for some $t>0$, every solution $u \in W^{2, p}$ of $L u=f$ has the form $u=e^{-\min \left(s_{0}, t\right) \rho} v$ for some $v \in W^{2, p}$.

Proof. Since the set of surjective maps is open in $\mathcal{L}\left(W^{2, p}, L^{p}\right)$, it follows from Lemma 2.1 that there is $s_{0}>0$ such that $L_{s}$ is onto $L^{p}$ for $0 \leq s \leq s_{0}$. By the local constancy of the index, $L_{s}$ is also Fredholm of index $\nu$ for $0 \leq s \leq s_{0}$ after shrinking $s_{0}$ if necessary. Thus, if $0 \leq s \leq s_{0}$, both $L$ and $L_{s}$ are onto $L^{p}$ and have $\nu$-dimensional null-space.

From now on, we choose $s:=\min \left(s_{0}, t\right)$ above. Obviously, $e^{s \rho} f \in L^{p}$ since $\rho \geq 0$. The solution set of $L_{s} v=e^{s \rho} f \in L^{p}$ in $W^{2, p}$ is a $\nu$-dimensional affine subspace $S_{s}$ of $W^{2, p}$. By (1.2), the equation $L_{s} v=e^{s \rho} f$ also reads $L\left(e^{-s \rho} v\right)=f$, and since the multiplication by $e^{-s \rho} \in W^{2, \infty}$ (by (2.2) and $\rho \geq 0$ ) is linear and one-to-one, it transforms the affine space $S_{s}$ into an affine space $S \subset W^{2, p}$ with the same 
dimension $\nu$. Every $u \in S$ has the form $u=e^{-s \rho} v$ and solves $L u=f$. On the other hand, by the same arguments as above, the whole solution set of $L u=f$ in $W^{2, p}$ is also a $\nu$-dimensional affine subspace of $W^{2, p}$ and therefore must coincide with $S$. This completes the proof.

The proof of the analog of Theorem 2.2 when $L$ is injective is a little more subtle:

Theorem 2.3. Let $p \in(1, \infty)$ be given. Suppose that $L \in \mathcal{L}\left(W^{2, p}, L^{p}\right)$ is Fredholm and injective (hence of index $\nu \leq 0$ ). Then, there is $s_{0}>0$ such that whenever $f \in L^{p}$ satisfies $e^{t \rho} f \in L^{p}$ for some $t>0$, the solution $u \in W^{2, p}$ of $L u=f$, if any, has the form $u=e^{-\min \left(s_{0}, t\right) \rho} v$ for some $v \in W^{2, p}$.

Proof. It follows once again from Lemma 2.1 and the local constancy of the index that $L_{s}$ is Fredholm of index $\nu$ for $0 \leq s \leq s_{0}$ and $s_{0}>0$ small enough. Furthermore, after shrinking $s_{0}>0$ if necessary, the nullity of $L_{s}$ cannot exceed the nullity of $L$ ([22, Theorem 5.17, p. 235], [24, Proposition 2.c.9, p. 78]). Since ker $L=\{0\}$, both $L$ and $L_{s}$ are injective and thus have a $|\nu|$-codimensional range if $0 \leq s \leq s_{0}$.

From now on, we choose $s:=\min \left(s_{0}, t\right)$ above. Let $G_{s} \subset L^{p}$ be a $|\nu|$-dimensional complement of rge $L_{s}$. Given $\varphi \in C_{0}^{\infty}$, we have $e^{s \rho} \varphi \in L^{p}$, and hence there is $\gamma_{s} \in G_{s}$ such that $e^{s \rho} \varphi+\gamma_{s} \in \operatorname{rge} L_{s}$. Let $\psi \in W^{2, p}$ be such that $L_{s} \psi=e^{s \rho} \varphi+\gamma_{s}$, so that $L\left(e^{-s \rho} \psi\right)=\varphi+e^{-s \rho} \gamma_{s}$. Denote by $G$ the space $G:=e^{-s \rho} G_{s} \subset L^{p}$. Clearly, $\operatorname{dim} G=\operatorname{dim} G_{s}=|\nu|$, and the relation $L\left(e^{-s \rho} \psi\right)=\varphi+e^{-s \rho} \gamma_{s}$ above shows that for every $\varphi \in C_{0}^{\infty}$ there is $\gamma \in G$ such that $\varphi+\gamma \in \operatorname{rge} L$. In other words, $C_{0}^{\infty} \subset$ $(\operatorname{rge} L)+G$. Since $\operatorname{rge} L$ is closed and $G$ is finite dimensional, the space (rge $L)+G$ is closed in $L^{p}$, and then the denseness of $C_{0}^{\infty}$ in $L^{p}$ yields that $L^{p}=(\operatorname{rge} L)+G$. Furthermore, since codim $\operatorname{rge} L=\operatorname{dim} G=|\nu|$, the sum must be direct, i.e., $G \cap$ rge $L=\{0\}$.

Now, since $e^{t \rho} f \in L^{p}$, then $e^{s \rho} f \in L^{p}$. From the above, there are $v \in W^{2, p}$ and $g_{s} \in G_{s}$ such that $L_{s} v=e^{s \rho} f+g_{s}$, and then $L\left(e^{-s \rho} v\right)=f+g$, where $g:=e^{-s \rho} g_{s} \in G$. Since $L u=f$, this yields $L\left(e^{-s \rho} v-u\right)=g$. Thus, $g \in G \cap$ rge $L=\{0\}$ and hence $g=0$. But then, $L\left(e^{-s \rho} v-u\right)=0$, so that $u=e^{-s \rho} v$ since $L$ is injective.

\section{Decay of solutions in the Fredholm CASE}

Some strengthening of our previous assumptions will be needed here: The coefficients $a_{i j}$ are real and locally Lipschitz continuous. As before, $b_{i}, c \in L^{\infty}$ are complex-valued. In addition, we assume that the local ellipticity condition

$$
\sum_{i, j=1}^{N} a_{i j}(x) \eta_{i} \eta_{j} \geq \gamma(x)|\eta|^{2}, \quad \forall \eta=\left(\eta_{1}, \ldots, \eta_{N}\right) \in \mathbb{R}^{N}
$$

holds, where $\gamma: \mathbb{R}^{N} \rightarrow(0, \infty)$ is bounded from below by a positive constant on every compact subset of $\mathbb{R}^{N}$.

With $p \in(1, \infty)$ being fixed, the approximation theorem of this section asserts that if $L \in \mathcal{L}\left(W^{2, p}, L^{p}\right)$ is Fredholm of index $\nu$, there is $d \in C_{0}^{\infty}$ such that $L+d$ is surjective if $\nu \geq 0$ and injective if $\nu \leq 0$, where of course $d$ is identified with a multiplication operator.

We shall make use the following property of the null-functions of $L$, which is a by-product of unique continuation and for which the stronger assumptions about the coefficients $a_{i j}$ made above are needed. 
Lemma 3.1. Let $\phi \in \operatorname{ker} L \subset W^{2, p}$. Then, either $\phi=0$ or $\phi^{-1}(0)$ has Lebesgue measure 0 .

There are various sources for Lemma 3.1. First, note that it is not restrictive to assume $a_{i j}=a_{j i}$ and that since $a_{i j} \in W_{l o c}^{1, \infty}, L$ can be written in the form (used in all the references)

$$
L=-\sum_{i, j=1}^{N} \partial_{j}\left(a_{i j}(x) \partial_{i}\right)+\sum_{i=1}^{N} b_{i}^{\prime}(x) \partial_{i}+c(x),
$$

with $b_{i}^{\prime} \in L^{\infty}$. If $N \geq 3$, Lemma 3.1 is in Garofalo and Lin [17 p. 352]. For $N \geq 2$, Robbiano [30] proves the sharper result that the Hausdorff dimension of $\phi^{-1}(0)$ is at most $N-1$, generalizing a result of Caffarelli and Friedman [9] when $a_{i j}=\delta_{i j}$. Other proofs in special cases are given by Gossez and Loulit 19 and de Figueiredo and Gossez 16. The case $N=1$ must be discussed separately. A complete proof when $N \geq 1$ can be found in [26] 4

If $p \in(1, \infty)$ and $L \in \mathcal{L}\left(W^{2, p}, L^{p}\right)$ is Fredholm, we let $Q$ denote an arbitrarily chosen continuous projection onto rge $L$. Then, $P=I-Q$ projects onto some finite dimensional complement of rge $L$. Given $\theta \in C_{0}^{\infty}$ (to be specified later), we identify $\theta$ with the (linear, continuous) operator of multiplication by $\theta$ from $W^{2, p}$ to $L^{p}$. Then, the operators $P \theta$ and $Q \theta$ are well defined and continuous from $W^{2, p}$ to $L^{p}$. Irrespective of the choice of $\theta$, we have

Lemma 3.2. Let $p \in(1, \infty)$ and assume that $L \in \mathcal{L}\left(W^{2, p}, L^{p}\right)$ is Fredholm. Given $\theta \in C_{0}^{\infty}$, the inequality

$$
\operatorname{dim} \operatorname{ker}(L+t \theta) \leq \operatorname{dim} \operatorname{ker}(P \theta)_{\mid \operatorname{ker} L}
$$

holds for $|t|>0$ small enough 5

Proof. Choose a closed complement $V \subset W^{2, p}$ of ker $L$. For $u \in \operatorname{ker}(L+t \theta) \subset$ $W^{2, p}$, write $u=z+v$ with $z \in \operatorname{ker} L$ and $v \in V$. Then, $(L+t \theta) u=0$ reads $(L+t \theta)(z+v)=0$; that is, $t \theta(z+v)+L v=0$, since $L z=0$. For $t \neq 0$ and since $P L=0$, this amounts to

$$
\left\{\begin{array}{c}
P \theta(z+v)=0 \\
Q(L+t \theta) v=-t Q \theta z .
\end{array}\right.
$$

Now, $Q L$ is an isomorphism of $V$ onto $\operatorname{rge} L$, and hence $Q(L+t \theta)$ remains an isomorphism of $V$ onto $\operatorname{rge} L$ for $|t|>0$ small enough. Thus, the second relation in (3.3) yields $v=-t[Q(L+t \theta)]^{-1} Q \theta z$. By substitution, we find that $u \in \operatorname{ker}(L+t \theta)$ if and only if $P \theta\left(I-t[Q(L+t \theta)]^{-1} Q \theta\right) z=0$ provided that $|t|>0$ is small enough, and then $u=z-t[Q(L+t \theta)]^{-1} Q \theta z$, a one-to-one function of $z \in \operatorname{ker} L$. Thus,

$$
\operatorname{dim} \operatorname{ker}(L+t \theta)=\operatorname{dim} \operatorname{ker} P \theta\left(I-t[Q(L+t \theta)]^{-1} Q \theta\right)_{\mid \operatorname{ker} L}
$$

for small $|t|>0$. Since $P \theta\left(I-t[Q(L+t \theta)]^{-1} Q \theta\right)_{\mid \operatorname{ker} L}$ is a finite dimensional operator, the dimension of its null space can only decrease in the vicinity of $t=0$. Thus, dim $\operatorname{ker} P \theta\left(I-t[Q(L+t \theta)]^{-1} Q \theta\right)_{\mid \operatorname{ker} L} \leq \operatorname{dim} \operatorname{ker}(P \theta)_{\mid \operatorname{ker} L}$ for $|t|>0$ small enough, whence (3.2) holds.

\footnotetext{
${ }^{4}$ The assumption that $a_{i j} \in W^{1, \infty}$ and the uniform elipticity in that reference are not needed for this particular result.

${ }^{5}$ But usually breaks down when $t=0$.
} 
Theorem 3.3. Let $p \in(1, \infty)$ and assume that $L \in \mathcal{L}\left(W^{2, p}, L^{p}\right)$ is Fredholm of index $\nu$. If $\nu \geq 0$ (resp. $\nu \leq 0$ ), there is a function $d \in C_{0}^{\infty}$ such that $L+d$ $\in \mathcal{L}\left(W^{2, p}, L^{p}\right)$ is surjective (resp. injective).

Proof. Assume first that $\nu \geq 0$. If $L$ is surjective, choose $d=0$. Otherwise, let $r:=$ codim $\operatorname{rge} L \geq 1$, so that $k:=\operatorname{dim} \operatorname{ker} L=\nu+r \geq r \geq 1$ and $k>\nu \geq 0$. It suffices to show that there is a function $d_{1} \in C_{0}^{\infty}$ such that $\operatorname{dim} \operatorname{ker}\left(L+d_{1}\right)<k$. Indeed, the multiplication by $d_{1}$ (with compact support) is a compact operator from $W^{2, p}$ to $L^{p}$, so that the operator $L+d_{1}$ remains Fredholm of index $\nu$ from $W^{2, p}$ to $L^{p}$. In particular, $\operatorname{dim} \operatorname{ker}\left(L+d_{1}\right) \geq \nu$. If $\operatorname{dim} \operatorname{ker}\left(L+d_{1}\right)=\nu$, then codim $\operatorname{rge}\left(L+d_{1}\right)=0$, whence $L+d_{1}$ is surjective and we may choose $d=d_{1}$. If $\operatorname{dim} \operatorname{ker}\left(L+d_{1}\right)>\nu$, the relations $\operatorname{dim} \operatorname{ker}\left(L+d_{1}\right)-$ codim $\operatorname{rge}\left(L+d_{1}\right)=\nu=k-r$ and $\operatorname{dim} \operatorname{ker}\left(L+d_{1}\right)<k$ show that $1 \leq \operatorname{codim} \operatorname{rge}\left(L+d_{1}\right)<r$. Thus, even though $d_{1}$ does not solve the problem, still passing from $L$ to $L+d_{1}$ decreases the codimension of the range. Since $L+d_{1}$ is a differential operator with the same properties as $L$, the procedure can be repeated with $L$ replaced by $L+d_{1}$. After a number $\ell$ of steps with $\ell \leq r$, we obtain functions $d_{1}, \ldots, d_{\ell}$ in $C_{0}^{\infty}$ such that $L+d$ is surjective, where $d:=d_{1}+\ldots+d_{\ell}$.

To find $d_{1}$, choose an arbitrary complement $\operatorname{span}\left\{\psi_{1}, \ldots, \psi_{r}\right\}$ of $\operatorname{rge} L$, with $\psi_{i} \in$ $L^{p}$ linearly independent. Since rge $L=\left(\operatorname{ker} L^{*}\right)^{\perp}$, where $L^{*} \in \mathcal{L}\left(L^{p^{\prime}}, W^{-2, p^{\prime}}\right)$ is the adjoint of $L\left(p^{\prime}:=p /(p-1)\right)$, there is a basis $\left\{\psi_{1}^{*}, \ldots, \psi_{r}^{*}\right\}$ of $\operatorname{ker} L^{*} \subset L^{p^{\prime}}$ such that $\int_{\mathbb{R}^{N}} \psi_{i} \psi_{j}^{*}=\delta_{i j}$ (Kronecker delta). The projection $P$ onto $\operatorname{span}\left\{\psi_{1}, \ldots, \psi_{r}\right\}$ along rge $L$ is given by

$$
P f=\sum_{i=1}^{r}\left(\int_{\mathbb{R}^{N}} f \psi_{i}^{*}\right) \psi_{i}, \quad \forall f \in L^{p} .
$$

Now, let $\left\{\phi_{1}, \ldots, \phi_{k}\right\} \subset W^{2, p}$ be some basis of ker $L$. By (3.4), the $r \times k$ matrix $M_{\theta}$ of $(P \theta)_{\mid \operatorname{ker} L}$ in the bases $\left\{\phi_{1}, \ldots, \phi_{k}\right\}$ and $\left\{\psi_{1}, \ldots, \psi_{r}\right\}$ has coefficients $\int_{\mathbb{R}^{N}} \theta \phi_{j} \psi_{i}^{*}$. We claim that there is an open ball $B \subset \mathbb{R}^{N}$ such that $\phi_{1} \psi_{1}^{*} \in L^{1}(B) \backslash\{0\}$. Indeed, if $B$ does not exist, then $\phi_{1} \psi_{1}^{*}=0$ a.e. on $\mathbb{R}^{N}$. Since $\phi_{1} \neq 0$, it follows from Lemma 3.1 that $\phi_{1}^{-1}(0)$ has Lebesgue measure 0 , whence $\psi_{1}^{*}=0$ a.e., which contradicts $\psi_{1}^{*} \neq 0$ in $L^{p^{\prime}}$. Thus, $B$ exists.

Since $\phi_{1} \psi_{1}^{*} \in L^{1}(B) \backslash\{0\}$, there is $\theta \in C_{0}^{\infty}(B)$ such that $\int_{\mathbb{R}^{N}} \theta \phi_{1} \psi_{1}^{*}=\int_{B} \theta \phi_{1} \psi_{1}^{*} \neq$ 0 . From the above, $M_{\theta} \neq 0$, whence $\operatorname{dim} \operatorname{ker} M_{\theta} \leq k-1$ since $M_{\theta}$ is $r \times k$; that is, $\operatorname{dim} \operatorname{ker}(P \theta)_{\mid \operatorname{ker} L} \leq k-1$. By Lemma 3.2 it follows that $\operatorname{dim} \operatorname{ker}(L+t \theta) \leq k-1$ for $|t|>0$ small enough, so that $d_{1}=t \theta$ with $|t|>0$ small enough works.

If now $\nu \leq 0$, the procedure is entirely similar: If $L$ is injective, let $d=0$. Otherwise, $k:=\operatorname{dim} \operatorname{ker} L \geq 1$ and $r:=$ codim $\operatorname{rge} L=k-\nu \geq k \geq 1$ since $\nu \leq 0$. As above, there is $d_{1} \in C_{0}^{\infty}$ such that $\operatorname{dim} \operatorname{ker}\left(L+d_{1}\right)<k$, and after $\ell$ steps with $\ell \leq k$, we obtain functions $d_{1}, \ldots, d_{\ell}$ in $C_{0}^{\infty}$ such that $L+d$ is injective, where $d:=d_{1}+\ldots+d_{\ell}$.

In Theorem 3.3, $\theta$ may also be assumed to be real valued if desired. When $p=2$ and $L$ is selfadjoint (hence of index 0 ), a simpler "abstract" proof of Theorem 3.3 is given in [28, Lemma 8], which does not use unique continuation.

The main result of this paper, Theorem 3.4 below, follows at once from Theorems 2.2, 2.3 and 3.3 . The $C^{2}$ function $\rho \geq 0$ is the same as in the previous section (so that (2.2) holds). 
Theorem 3.4. Let $p \in(1, \infty)$ be given. Suppose that $L \in \mathcal{L}\left(W^{2, p}, L^{p}\right)$ is Fredholm. Then, there is $s_{0}>0$ such that whenever $f \in L^{p}$ satisfies $e^{t \rho} f \in L^{p}$ for some $t>0$, every solution $u \in W^{2, p}$ of $L u=f$ has the form $u=e^{-\min \left(s_{0}, t\right) \rho} v$ for some $v \in W^{2, p}$.

Proof. Let $\nu \in \mathbb{Z}$ denote the index of $L$. If $\nu \geq 0$, Theorem 3.3 yields $d \in C_{0}^{\infty}$ such that $L+d$ is surjective. Of course, $L+d \in \mathcal{L}\left(W^{2, p}, L^{p}\right)$ is a compact perturbation of $L$, hence Fredholm, and it satisfies the conditions required in Theorem 2.2. Rewrite $L u=f$ as $(L+d) u=f+d u$. Since $e^{t \rho} f \in L^{p}$ and $d$ has compact support, we have $e^{t \rho}(f+d u) \in L^{p}$, and the conclusion follows from Theorem 2.2 for $L+d$.

If $\nu \leq 0$, Theorem 3.3 yields $d \in C_{0}^{\infty}$ such that $L+d$ is injective. The above arguments can then be repeated with Theorem 2.3 replacing Theorem 2.2

As noted in the Introduction, (2.2) holds if $\rho(x)=|x|$ for $|x|$ large enough, and then the relations $e^{t \rho} f \in L^{p}$ and $u=e^{-\min \left(s_{0}, t\right) \rho} v$ in Theorem 3.4 are simply $e^{t|x|} f \in L^{p}$ and $u=e^{-\min \left(s_{0}, t\right)|x|} v$ for large $|x|$. If $\rho(x)=\log |x|$ for $|x|$ large enough, then once again (2.2) holds, and then $|x|^{t} f \in L^{p}$ while $u=|x|^{-\min \left(s_{0}, t\right)} v$ for $|x|$ large enough in Theorem 3.4. Of course, $\rho(x)=|x|^{\alpha}$ for large $|x|$ does not satisfy (2.2) if $\alpha>1$. In fact, the boundedness of $D \rho$ on $\mathbb{R}^{N}$ and the mean value theorem show that Theorem 3.4 cannot deliver more than exponential decay.

Remark 3.1. If $e^{-\tau \rho} \in \bigcap_{1 \leq q \leq \infty} W^{2, q}$ for every $\tau>0$ (for instance if $\rho(x)=|x|$ for $|x|$ large enough), then in particular $e^{-\min \left(s_{0}, t\right) \rho} \in \bigcap_{p^{\prime} \leq q \leq \infty} W^{2, q}$ with $p^{\prime}:=$ $p /(p-1)$ and $u=e^{-\min \left(s_{0}, t\right) \rho} v$ in Theorem 3.4 is in $\bigcap_{1 \leq r \leq p} W^{2, r}$. This is consistent with the fact that $e^{t \rho} f \in L^{p}$ implies $f \in \bigcap_{1 \leq r \leq p} L^{r}$, but cannot be deduced from elliptic regularity: The ellipticity condition (3.1) is not strong enough to ensure that $f \in L^{r}$ implies $u \in W^{2, r}$ for $r \in(1, p)$, and, in addition, no regularity result exists under any assumption for $r=1$.

\section{EXPONENTIAL DECAY OF THE GENERALIZED EIGENFUNCTIONS}

The assumptions about the coefficients of $L$ are the same as in the previous section. If $p \in(1, \infty)$, then $L \in \mathcal{L}\left(W^{2, p}, L^{p}\right)$ and $L$ may also be viewed as an unbounded operator on $L^{p}$ with domain $W^{2, p}$, so that the concept of eigenvalue of $L$ makes sense. As noted in the Introduction, the existence of $\lambda \in \mathbb{C}$ such that $L-\lambda$ is Fredholm implies that $L$ must also be closed.

If $\lambda \in \mathbb{C}$ is an eigenvalue of $L$, a generalized eigenfunction of $L$ associated with $\lambda$ is any function $u \in W^{2, p}$ such that $(L-\lambda)^{j} u \in W^{2, p}$ for $1 \leq j \leq k-1$ and $(L-\lambda)^{k} u=0$ for some integer $k \in \mathbb{N}$. Recall that a Fredholm eigenvalue of $L$ was defined as an eigenvalue $\lambda$ such that $L-\lambda \in \mathcal{L}\left(W^{2, p}, L^{p}\right)$ is Fredholm. With this definition, the exponential decay of the generalized eigenfunctions is a straightforward corollary to Theorem 3.4.

Theorem 4.1. Let $\rho \geq 0$ be a $C^{2}$ function such that $\rho(x)=|x|$ for $|x|$ large enough. Let $p \in(1, \infty)$ be given and let $\lambda$ be a Fredholm eigenvalue of $L$ viewed as an unbounded operator on $L^{p}$ with domain $W^{2, p}$. Then, there is $s_{\lambda}>0$, independent of $\rho$, such that every generalized eigenfunction $u$ associated with $\lambda$ has the form $u=e^{-s_{\lambda} \rho} v$ for some $v \in W^{2, p}$.

Proof. The function $\rho$ satisfies (2.2). By changing $L$ into $L-\lambda$, the problem is reduced to the case $\lambda=0$. Let $s_{0}>0$ be given by Theorem 3.4 If $u \in \operatorname{ker} L$, then 
Theorem 3.4 with $f=0$ and $t=s_{0}$ shows that $u=e^{-s_{0} \rho} v$ with $v \in W^{2, p}$. Next, let $u \in \operatorname{ker} L^{2}$, so that $L u=u_{1} \in \operatorname{ker} L$ and hence $L u=e^{-s_{0} \rho} v_{1}$ with $v_{1} \in W^{2, p}$. Now, Theorem 3.4 with $f=e^{-s_{0} \rho} v_{1}$ and $t=s_{0}$ yields $u=e^{-s_{0} \rho} v$ with $v \in W^{2, p}$. By induction, the same argument shows that $u=e^{-s_{0} \rho} v$ with $v \in W^{2, p}$ whenever $L^{k} u=0$ for some integer $k \in \mathbb{N}$. Since two choices of $\rho$ coincide outside of a ball, it is obvious that $s_{0}$ does not depend upon $\rho$.

Remark 4.1. It follows from Remark 3.1 that the generalized eigenfunctions $u$ in Theorem 4.1 satisfy $u \in \bigcap_{1 \leq r \leq p} W^{2, r}$. If all the coefficients are real, $a_{i j} \in W^{1, \infty}$ and uniform ellipticity holds, then the generalized eigenfunctions associated with Fredholm eigenvalues of index 0 satisfy $u \in \bigcap_{1<q<\infty} W^{2, q}$ by the remarks about $p$ independence in the next section, whence $u \in \bigcap_{1 \leq q<\infty} W^{2, q}$. Then, by Theorem 4.1 with $p$ replaced by any $q>N$, it follows that $u$ and $\nabla u$ have pointwise exponential decay.

As noted in the Introduction, Fredholm eigenvalues of index $\nu \neq 0$ are not, and those of index 0 need not be, isolated eigenvalues. The case of isolated eigenvalues of finite multiplicity is discussed in Section [7, independently of Theorem 4.1 and based on a straightforward perturbation argument.

\section{Complements}

5.1. $p$-independence. A priori at least, everything in Theorem 3.4 depends upon $p$ : The assumption that $L$ is Fredholm, its index, and even $s_{0}$ and $v$. However, when all the coefficients of $L$ are real, $a_{i j} \in W^{1, \infty}$ and (3.1) is replaced by a stronger uniform ellipticity condition, it is shown in [26, Theorem 6.3] that if $L$ is Fredholm of index 0 for some $p \in(1, \infty)$, then it remains Fredholm of index 0 for all other values of $p$. This $p$-independence is still an open question when the coefficients $b_{i}$ and $c$ are complex or when $L$ has index $\nu \neq 0$.

When the above assumptions hold and hence the condition that $L$ is Fredholm of index 0 is independent of $p \in(1, \infty)$, a stronger $p$-independence result is also true. Let $s_{0}>0$ be given by Theorem 3.4 for a chosen value of $p \in(1, \infty)$ and suppose that $e^{t \rho} f \in L^{p} \cap L^{q}$ with $t>0$ and $q \in(1, \infty)$. Then, $u=e^{-\min \left(s_{0}, t\right) \rho} v$ for some $v \in W^{2, p} \cap W^{2, q}$. To see this, recall that the representation $u=e^{-s \rho} v$ with $s:=\min \left(s_{0}, t\right)$ and $v \in W^{2, p}$ was obtained in the proof of Theorem 3.4 by writing $L u=f$ as $(L+d) u=f+d u$, where $d \in C_{0}^{\infty}$ is such that $L+d$ is surjective, and by choosing $s_{0}>0$ such that $(L+d)_{s}=L_{s}+d$ is Fredholm of index 0 and surjective for $0 \leq s \leq s_{0}$. Since $L_{s}+d$ has index 0 , surjectivity amounts to invertibility, and then $u=e^{-s \rho} v$, where $v \in W^{2, p}$ is the (unique) solution of $\left(L_{s}+d\right) v=e^{s \rho}(f+d u)$. Now, since $e^{t \rho} f \in L^{p} \bigcap L^{q}$ implies $f \in L^{p} \cap L^{q}$ (recall $\rho \geq 0$ ) and since $L u=f$ and $L$ is Fredholm of index 0, [26, Theorem 7.1] ensures that $u \in W^{2, p} \cap W^{2, q}$ (if $q \leq p$ and $e^{-s \rho} \in \bigcap_{1 \leq q \leq \infty} W^{2, q}$ for every $s>0$, this also follows from Remark 3.1). Therefore, $e^{s \rho} d u \in L^{p} \cap L^{q}$, and hence $e^{s \rho}(f+d u) \in L^{p} \cap L^{q}$. By using once again [26, Theorem 7.1], but now with $L_{s}+d$, we get $v \in W^{2, p} \cap W^{2, q}$, as claimed. (Since the second order coefficients of $L_{s}$ and $L$ are the same, [26. Theorem 7.1] can be used with $L_{s}+d$.) 
5.2. Generalizations. Theorems 2.2 and 2.3 require little more than the fact that $L$ is a differential operator, and they can be generalized 6 to other unbounded domains, higher order equations or systems. The generalization of Theorems 3.3 and 3.4 to various other unbounded domains (with boundary conditions), in particular cylindrical ones, is routine, but the case of higher order problems or systems is more delicate because the proof of Theorem 3.3 uses the unique continuation property via Lemma 3.1. However, some related material is available, though not always in the (needed) sharpest form. See for instance Alinhac and Baouendi [3], Anane et al. 5], De Carli 13], 14, Dehman and Robbiano [15, Wang 35] and the references therein.

The question whether Theorem 3.4 remains true when $L$ is semi-Fredholm (so that its index may be $\infty$ or $-\infty$ ) is open. Difficulties arise with the proof of Theorem 3.3 notably the possible lack of closed complements for $\operatorname{ker} L$ (if $\nu=\infty$ ) or for rge $L$ (if $\nu=-\infty$ ) when $p \neq 2$, and others with the proofs of Theorems 2.2 and 2.3. where finite dimensionality arguments are involved.

5.3. Examples with nonzero index. There is of course no difficulty in finding operators $L$ in (1.1) which are Fredholm of index 0 from $W^{2, p}$ to $L^{p}$. Simple examples also exist with index $\nu \neq 0$. With $N=1$, let

$$
L:=-u^{\prime \prime}-b(x) u^{\prime}-2 u,
$$

where $b$ is a smooth function such that $b(x)=2$ for $x \geq 1$ and $b(x)=-2$ for $x \leq-1$. By elementary ODE theory, $L \in \mathcal{L}\left(W^{2, p}, L^{p}\right)(p \in(1, \infty))$ has a twodimensional null space: First, $L$ vanishes on a two-dimensional subspace of $C^{\infty}$. Next, if $u \in C^{\infty}$ and $L u=0$, then $u(x)$ is a linear combination of $e^{-x} \cos x$ and $e^{-x} \sin x$ for $x \geq 1$ and a linear combination of $e^{x} \cos x$ and $e^{x} \sin x$ for $x \leq-1$. Observe in passing that the exponential decay of $u$ at infinity is consistent with Theorem 4.1 That $L$ is onto $L^{p}$, hence $L$ has index 2 , follows from the "variation of parameters" formula and well known properties of convolution: Let $\left\{u_{1}, u_{2}\right\}$ be a basis of $\operatorname{ker} L \subset W^{2, p}$ and let $f \in L^{p}$. Then,

$$
u(x)=\left(-\int_{0}^{x} \frac{f u_{2}}{w}\right) u_{1}(x)+\left(\int_{0}^{x} \frac{f u_{1}}{w}\right) u_{2}(x),
$$

with $w$ the Wronskian of $u_{1}$ and $u_{2}$, solves $L u=f$. To show that $u \in L^{p}$, it suffices to show that $u \in L^{p}(0, \infty)$ and $u \in L^{p}(-\infty, 0)$. We just prove the former relation. On $(0, \infty)$, the functions $u_{i}, i=1,2$, have the form $u_{i}(x)=e^{-x} v_{i}(x)$ with $v_{i}$ continuous on $[0, \infty)$ and $v_{i}(x)$ a linear combination of $\sin x$ and $\cos x$ for $x \geq 1$. Thus, $v_{i} \in L^{\infty}(0, \infty)$. Likewise, $w(x)=e^{2 x} w_{0}(x)$, where $w_{0}$ is a continuous nonvanishing function on $[0, \infty)$ and $w_{0}(x)=1$ for $x \geq 1$. Thus, $z_{i}:=v_{i} / w_{0} \in$ $L^{\infty}(0, \infty)$ and, by (5.2),

$$
u=-\left(\tilde{f}_{2} * \tilde{e}\right) v_{1}+\left(\tilde{f}_{1} * \tilde{e}\right) v_{2} \text { on }(0, \infty)
$$

where $\tilde{f}_{i}:=f(x) z_{i}(x)$ if $x \geq 0$ and 0 otherwise, and $\tilde{e}(x):=e^{-x}$ if $x \geq 0$ and 0 otherwise. Obviously, $\tilde{f}_{i} \in L^{p}$ and $\tilde{e} \in L^{1}$, so that $\tilde{f}_{i} * \tilde{e} \in L^{p}$ and hence $u \in L^{p}(0, \infty)$. Similar arguments yield $u \in L^{p}(-\infty, 0)$, so that $u \in L^{p}$. By differentiation of (5.2) and the same procedure, $u^{\prime} \in L^{p}$, and then $u \in W^{2, p}$ is obvious.

\footnotetext{
${ }^{6}$ However, recall the hypothesis that $L$ is Fredholm places implicit limitations.
} 
Observe that Theorem 4.1 is corroborated by the representation (5.2): If $f=$ $e^{-t|x|} g$ with $t>0$ and $g \in L^{p}$, then $u$ decays exponentially like $e^{-\min (1, t)|x|}$ if $t \neq 1$ and like $|x|^{1-\frac{1}{p}} e^{-|x|}$ if $t=1$. Thus, in all cases, $u$ decays exponentially like $e^{-\min \left(s_{0}, t\right)|x|}$ for any $s_{0}<1$.

The formal adjoint of $L$ in (5.1), that is,

$$
L^{*} u:=-u^{\prime \prime}+b(x) u^{\prime}+\left(b^{\prime}(x)-2\right) u,
$$

has index -2 . Indeed, with $p^{\prime}$ (Hölder conjugate of $p$ ) replacing $p$ above, $L$ is Fredholm of index 2 and surjective from $W^{2, p^{\prime}}$ to $L^{p^{\prime}}$ and $L^{*} \in \mathcal{L}\left(L^{p}, W^{-2, p}\right)$ is the actual adjoint of $L$ in this setting. Thus, $L^{*} \in \mathcal{L}\left(L^{p}, W^{-2, p}\right)$ has index -2 and is injective on $L^{p}$. Therefore, $L^{*}$ is injective on $W^{2, p} \subset L^{p}$. Since $L^{*}\left(L^{p}\right) \subset W^{-2, p}$ has codimension 2 and since $C_{0}^{\infty}$ is dense in $W^{-2, p}$, there is a subspace $Z \subset C_{0}^{\infty}$ with $\operatorname{dim} Z=2$ such that $L^{*}\left(L^{p}\right) \cap Z=\{0\}$. In particular, $L^{*}\left(W^{2, p}\right) \cap Z=\{0\}$, and since $Z \subset L^{p}$, it follows that the codimension of $L^{*}\left(W^{2, p}\right)$ in $L^{p}$ is at least 2 . Now, for every $f \in W^{-2, p}$, the equation $L^{*} u=f+\varphi$ is solvable in $L^{p}$ for some $\varphi \in Z$. In particular, this is true if $f \in L^{p}$, and in that case it is easily seen that $u \in W^{2, p}$. This shows that the codimension of $L^{*}\left(W^{2, p}\right)$ in $L^{p}$ is at most 2 and hence equal to 2 . As a result, $L^{*} \in \mathcal{L}\left(W^{2 p}, L^{p}\right)$ is Fredholm of index -2 .

5.4. A counterexample to the exponential decay of eigenfunctions. It is well known that ellipticity alone does not ensure the exponential decay property of Theorem 4.1, even for operators with constant coefficients. For example, $u(x)=$ $J_{0}(|x|)$, where $J_{0}$ denotes the Bessel function of the first kind and index 0 , solves Helmholtz' equation $-\Delta u-u=0$ on $\mathbb{R}^{2}$ (Dautray and Lions [12 Vol. 1, p. 642]). From the well-known asymptotic behavior of Bessel functions, $u$ is continuous (even analytic) on $\mathbb{R}^{2}$ and $|u(x)| \sim \sqrt{\frac{2}{\pi}}|x|^{-\frac{1}{2}} \cos \left(|x|-\frac{\pi}{4}\right)$ for large $|x|$. Thus, $u \in L^{p}$ if $p>4$, and $u$ does not have exponential decay. Yet, $u \in W^{2, p}$ since $-\Delta u-u=0$ also reads $-\Delta u+u=2 u \in L^{p}$ (see 12 , Vol. 1 , Proposition 27, p. 635]). In this example, $-\Delta-1$ is not Fredholm. In fact, since not only $u$ above but also its translates (that is, $u(x+a)$ for $\left.a \in \mathbb{R}^{2}\right)$ are in $\operatorname{ker}(-\Delta-1)$, it follows that $\operatorname{ker}(-\Delta-1)$ is infinite dimensional in $W^{2, p}$ if $p>4$. More generally, all the radially symmetric nontrivial solutions $u$ of $-\Delta u-k^{2} u=0(k>0)$ provide counterexamples to the exponential decay in every dimension $N \geq 2$. (These solutions are in $W^{2, p}$ for $p>\frac{2 N}{N-1}$.)

\section{Application to quasilinear Problems}

We now discuss the decay of the solutions $u \in W^{2, p}$ of an equation $F(u)=f$ when

$$
F(u):=\sum_{i, j=1}^{N} a_{i j}(x, u) \partial_{i j}^{2} u+b(x, u, \nabla u) .
$$

Our assumptions about the coefficients $a_{i j}$ and $b$ involve the concept of equicontinuous $C_{\xi}^{k}$ bundle map ([29]), defined below. The definition is technical, but its purpose is simply to ensure that the Nemytskii operators associated with the coefficients $a_{i j}$ and $b$ in (6.1) map into the "right" spaces and give $F$ the necessary smoothness while not placing growth limitations on the nonlinearities.

Definition 6.1. The mapping $g: \mathbb{R}^{N} \times \mathbb{R}^{N+1} \rightarrow \mathbb{R}$ is said to be an equicontinuous $C^{0}$ bundle map if it is continuous and the collection $(g(x, \cdot))_{x \in \mathbb{R}^{N}}$ is equicontinuous 
at every point $\xi \in \mathbb{R}^{N+1}$. If $k \in \mathbb{N}, g$ is said to be an equicontinuous $C_{\xi}^{k}$ bundle map if $D_{\xi}^{\kappa} g$ exists and is an equicontinuous $C^{0}$ bundle map for $|\kappa| \leq k$.

For example, a function $g(x, \xi)=g_{1}(x) g_{2}(\xi)$ with nonconstant $g_{2}$ is an equicontinuous $C^{0}$ bundle map if and only if $g_{1}$ is bounded and continuous and $g_{2}$ is continuous. If also $g_{2}$ is $C^{k}$, then $g$ is an equicontinuous $C_{\xi}^{k}$ bundle map (if $g_{2}$ is constant, then $g_{1}$ need not be bounded). In general, the concept of equicontinuous $C_{\xi}^{k}$ bundle map places no restriction on the growth with respect to the $\xi$ variable, which is important in the applications.

The notation $\xi=\left(\xi_{0}, \ldots, \xi_{N}\right)$ is used throughout for $\xi \in \mathbb{R}^{N+1}$. We shall assume that

(6.2) $\quad a_{i j}$ is $C^{1}$ in $\left(x, \xi_{0}\right)$ and a $C_{\xi}^{1}$ equicontinuous bundle map with

$$
a_{i j}(\cdot, 0) \in L^{\infty} \text { for } 1 \leq i, j \leq N,
$$

$\partial_{x_{\ell}} a_{i j}$ is a $C^{0}$ equicontinuous bundle map with

$$
\partial_{x_{\ell}} a_{i j}(\cdot, 0) \in L^{\infty} \text { for } 1 \leq i, j, \ell \leq N,
$$

$$
\sum_{i, j=1}^{N} a_{i j}\left(x, \xi_{0}\right) \eta_{i} \eta_{j} \geq \gamma\left(x, \xi_{0}\right)|\eta|^{2}, \quad \forall \eta=\left(\eta_{1}, \ldots, \eta_{N}\right) \in \mathbb{R}^{N},
$$

for every $x \in \mathbb{R}^{N}$, where $\gamma: \mathbb{R}^{N} \times \mathbb{R} \rightarrow(0, \infty)$ is bounded from below by a positive constant $\gamma_{\tilde{K}}$ on every compact subset $\tilde{K}$ of $\mathbb{R}^{N} \times \mathbb{R}$ (for instance, $\gamma$ lower semicontinuous),

$$
b(x, \xi)=\sum_{i=1}^{N} b_{i}(x, \xi) \xi_{i}+c(x, \xi) \xi_{0}
$$

where $b_{i}, c$ are equicontinuous $C^{0}$ bundle maps satisfying

$$
b_{i}(\cdot, 0), c(\cdot, 0) \in L^{\infty} \text { for } 1 \leq i \leq N .
$$

For instance, (6.5) and (6.6) hold if $b$ is a $C_{\xi}^{1}$ equicontinuous bundle map with $b(\cdot, 0)=0$ and $\partial_{\xi_{i}} b(\cdot, 0) \in L^{\infty}$ for $0 \leq i \leq N$ (see [29] Theorem 2.3]).

Remark 6.1. Definition 6.1 implies that the coefficients $a_{i j}, b_{i}$ and $c$ are real, but the results of this section are unchanged if $b_{i}$ and $c$ are complex. The definition of an equicontinuous $C^{0}$ bundle map has a trivial generalization to complex-valued functions depending upon $(x, \xi) \in \mathbb{R}^{N} \times \mathbb{C}^{N+1}$. Then, $C_{\xi}^{k}$ bundle maps can be defined analogously, where differentiation is understood in the real sense (holomorphy is not needed). However, the coefficients $a_{i j}$ must be real.

For $u \in W^{1, p}$, we denote by $L(u)$ the linear differential operator

$$
\begin{aligned}
L(u):= & \sum_{i, j=1}^{N} a_{i j}(x, u(x)) \partial_{i j}^{2}+\sum_{i=1}^{N} b_{i}(x, u(x), \nabla u(x)) \partial_{i} \\
& +c(x, u(x), \nabla u(x)),
\end{aligned}
$$

so that, formally at least for now, $F(u)=f$ may be rewritten as $L(u) u=f$. We need two lemmas. The first one shows that $L(u) u$ does make sense and hence equals $F(u)$. 
Lemma 6.1. If $u \in W^{2, p}$ with $p \in(N, \infty)$, the coefficients of $L(u)$ in (6.7) satisfy the conditions $a_{i j}(\cdot, u(\cdot)) \in W^{1, \infty}, b_{i}(\cdot, u(\cdot), \nabla u(\cdot)) \in L^{\infty}$ and $c(\cdot, u(\cdot), \nabla u(\cdot)) \in L^{\infty}$.

Proof. By [29, Theorem 2.2 (ii)] and the hypotheses made above about $a_{i j}, b_{i}$ and $c$, it follows that $a_{i j}(\cdot, u(\cdot)), b_{i}(\cdot, u(\cdot), \nabla u(\cdot)), c(\cdot, u(\cdot), \nabla u(\cdot)) \in L^{\infty}$. It remains only to show that $\partial_{\ell} a_{i j}(\cdot, u(\cdot)) \in L^{\infty}$ for $1 \leq \ell \leq N$. Since $a_{i j}$ and $u$ are $C^{1}$, the chain rule gives $\partial_{\ell} a_{i j}(\cdot, u(\cdot))=\left(\partial_{x_{\ell}} a_{i j}\right)(\cdot, u(\cdot))+\partial_{\xi_{0}} a_{i j}(\cdot, u(\cdot)) \partial_{\ell} u$. Once again by [29] Theorem 2.2 (ii)], we have $\left(\partial_{x_{\ell}} a_{i j}\right)(\cdot, u(\cdot)) \in L^{\infty}$ and $\partial_{\xi_{0}} a_{i j}(\cdot, u(\cdot)) \in L^{\infty}$. Since also $\partial_{\ell} u \in L^{\infty}$ (when $p>N$, functions of $W^{1, p}$ are continuous and tend uniformly to 0 at infinity), it follows that $\partial_{\ell} a_{i j}(\cdot, u(\cdot)) \in L^{\infty}$.

If $a_{i j}$ depends upon $\xi$ and not only $\xi_{0}$, it is no longer possible to obtain

$$
a_{i j}(\cdot, u(\cdot), \nabla u(\cdot)) \in W^{1, \infty}
$$

in Lemma 6.1 This is why $a_{i j}$ was assumed to depend on $\xi_{0}$ alone.

The next lemma has a major practical importance, since it shows that the verification that $L(u) \in \mathcal{L}\left(W^{2, p}, L^{p}\right)$ is a Fredholm operator needs only to be made with the one choice $u=0$.

Lemma 6.2. If $u \in W^{2, p}$ with $p \in(N, \infty)$, then $L(u)$ in (6.7) is Fredholm of index $\nu$ from $W^{2, p}$ to $L^{p}$ if and only if the same thing is true of $L(0)=$ $\sum_{i, j=1}^{N} a_{i j}(x, 0) \partial_{i j}^{2}+\sum_{i=1}^{N} b_{i}(x, 0) \partial_{i}+c(x, 0)$.

Proof. This is essentially proved in [29], though not for quite the same operator $L(u)$. Instead of giving an almost identical (and technical) proof, we reduce the problem to a case when the results of [29] can be used. Denote by $L_{0}(u)$ the differential operator

$$
L_{0}(u):=\sum_{i, j=1}^{N} a_{i j}(x, u(x)) \partial_{i j}^{2}+\sum_{i=1}^{N} b_{i}(x, 0) \partial_{i}+c(x, 0) .
$$

Then, $L(u)-L_{0}(u)$ is a compact operator from $W^{2, p}$ to $L^{p}$, because

$$
\begin{aligned}
\lim _{|x| \rightarrow \infty} & b_{i}(x, u(x), \nabla u(x))-b(x, 0) \\
\quad= & \lim _{|x| \rightarrow \infty} c(x, u(x), \nabla u(x))-c(x, 0)=0
\end{aligned}
$$

and the multiplication by a continuous function tending uniformly to 0 at infinity is compact from $W^{1, p}$ to $L^{p}$.

From the above, it suffices to show that $L_{0}(u)$ is Fredholm of index $\nu$ if and only if $L_{0}(0)=L(0)$ is Fredholm of index 0 . If the $a_{i j}$ do not depend upon $\xi_{0}$, then $L_{0}(u)=L_{0}(0)$ and there is nothing to prove. Otherwise, we introduce the nonlinear mapping $F_{0}(u):=L_{0}(u) u$. By [29] Lemma 3.2], $F_{0}$ is of class $C^{1}$ from $W^{2, p}$ to $L^{p}$, and by [29, Theorem 3.8], $D F_{0}(u)$ is Fredholm of index $\nu$ if and only if $D F_{0}(0)$ is Fredholm of index $\nu$. Now, the derivative $D F_{0}(u)$ is given by ([29, Lemma 3.2])

$$
D F_{0}(u) v=L_{0}(u) v+\sum_{i, j=1}^{N} \partial_{\xi_{0}} a_{i j}(\cdot, u(\cdot)) v \partial_{i j} u,
$$

so that $D F_{0}(0)=L_{0}(0)$. Note that $\partial_{\xi_{0}} a_{i j}(\cdot, u(\cdot)) \partial_{i j} u$ and $\partial_{\xi_{0}} a_{i j}(\cdot, 0) \partial_{i j} u$ are functions in $L^{p}$ since $\partial_{\xi_{0}} a_{i j}(\cdot, u(\cdot)), \partial_{\xi_{0}} a_{i j}(\cdot, 0) \in L^{\infty}$ (see the proof of Lemma 6.1) and that the multiplication by a given function of $L^{p}$ is a compact operator from $W^{2, p}$ to $L^{p}$ when $p>N / 2$. Thus, $L_{0}(u)$ is a compact perturbation of $D F_{0}(u)$. It then 
follows from the above that $L_{0}(u)$ and $L_{0}(0)$ are Fredholm of index $\nu$ simultaneously.

Remark 6.2. (i) If the coefficients $a_{i j}$ depend upon $\xi_{0}$, then $L(u)-L(0)$ is not compact, so that Lemma 6.2 does not follow from the stability of Fredholm operators under compact perturbation. In the above proof, this issue is hidden in the result from 29 that $D F_{0}(u)$ and $D F_{0}(0)$ are simultaneously Fredholm of index $\nu$. (ii) If the coefficients $a_{i j}$ do not depend upon $\xi_{0}$, then (6.2) and (6.3) may be replaced by the more general and simpler condition that $a_{i j} \in W_{l o c}^{1, \infty}$, and (6.4) is the same as (3.1). (iii) If the coefficients $a_{i j}$ do not depend upon $\xi_{0}$ and $b$ does not depend upon $\left(\xi_{1}, \ldots, \xi_{N}\right)$ (that is, $b(x, u, \nabla u)=b(x, u)$ is independent of $\left.\nabla u\right)$, then Lemmas 6.1 and 6.2 are true for $p \in(\max (1, N / 2), \infty)$, under the weaker assumption about $a_{i j}$ mentioned in (ii).

The $C^{2}$ function $\rho \geq 0$ in Theorem 6.3 and Corollary 6.4 below is the same as in Sections 2 and 3 .

Theorem 6.3. Let $p \in(N, \infty)$ be given and suppose that the nonlinear operator $F$ in (6.1) is Fredholm from $W^{2, p}$ to $L^{p}$. Let $f \in L^{p}$ be such that $e^{t \rho} f \in L^{p}$ for some $t>0$. Then, every solution $u \in W^{2, p}$ of $F(u)=f$ has the form $u=e^{-s \rho} v$ for some $s>0$ and some $v \in W^{2, p}$.

Proof. From 29, Lemma 3.2], $F$ is a well defined $C^{1}$ mapping from $W^{2, p}$ to $L^{p}$ with derivative $D F(0)=L(0)$. By Lemma 6.1 $F(u)=L(u) u$ with $L(u)$ given by 6.7). The assumption that $F$ is Fredholm entails that $D F(0)=L(0)$ is Fredholm, and the conclusion follows from Lemmas 6.1 and 6.2 and Theorem 3.4 for the operator $L(u)$.

By Remark 3.1 for $L(u)$, we have $u \in \bigcap_{1 \leq r \leq p} W^{2, r}$ in Theorem 6.3 if $e^{-\tau \rho} \in$ $\bigcap_{1 \leq q \leq \infty} W^{2, q}$ for every $\tau>0$.

In Theorem 6.3] $s$ depends upon $t$ and upon the solution $u$. In fact, the dependence on $u$ can be eliminated under an additional regularity assumption about $b$ :

Corollary 6.4. Suppose also that the coefficients $b_{i}$ and $c$ are $C_{\xi}^{1}$ bundle maps and that $\partial_{\xi_{j}} b_{i}(\cdot, 0) \in L^{\infty}$ and $\partial_{\xi_{j}} c(\cdot, 0) \in L^{\infty}$ for $0 \leq j \leq N$. Then, there is $s_{0}>0$ depending only on $D F(0)$ such that $s=\min \left(s_{0}, t\right)$ in Theorem 6.3.

Proof. Let $s_{0}$ be given by Theorem 3.4 for $L(0)=D F(0)$. Since the coefficients $a_{i j}, b_{i}$ and $c$ are $C_{\xi}^{1}$ bundle maps, we have

$$
\begin{gathered}
a_{i j}\left(x, \xi_{0}\right)-a_{i j}(x, 0)=\tilde{a}_{i j}\left(x, \xi_{0}\right) \xi_{0}, \\
b_{i}(x, \xi)-b_{i}(x, 0)=\tilde{b}_{i 0}(x, \xi) \xi_{0}+\sum_{\ell=1}^{N} \tilde{b}_{i \ell}(x, \xi) \xi_{\ell}, \\
c(x, \xi)-c(x, 0)=\tilde{c}_{0}(x, \xi) \xi_{0}+\sum_{\ell=1}^{N} \tilde{c}_{\ell}(x, \xi) \xi_{\ell},
\end{gathered}
$$

for some equicontinuous $C^{0}$ bundle maps $\tilde{a}_{i j}, \tilde{b}_{i \ell}$ and $\tilde{c}_{\ell}$ satisfying $\tilde{a}_{i j}(\cdot, 0) \in L^{\infty}$, $\tilde{b}_{i \ell}(\cdot, 0) \in L^{\infty}$ and $\tilde{c}_{\ell}(\cdot, 0) \in L^{\infty}$ for $0 \leq \ell \leq N$ (see [29, Theorem 2.3]). Then, $\tilde{a}_{i j}(\cdot, u) \in L^{\infty}, \tilde{b}_{i \ell}(\cdot, u, \nabla u) \in L^{\infty}$ and $\tilde{c}_{\ell}(\cdot, u, \nabla u) \in L^{\infty}$ by [29, Theorem 2.2 (i)].

\footnotetext{
${ }^{7}$ Which is equivalent to $D F(0)$ Fredholm by [29, Theorem 3.8].
} 
From the above and $F(u)=L(u) u$, we get

$$
\begin{aligned}
& L(0) u-F(u)=w_{0}(u) u^{2}+\sum_{i=1}^{N} w_{i}(u) u \partial_{i} u \\
& \quad+\sum_{i, j=1}^{N} w_{i j}(u) \partial_{i} u \partial_{j} u+\sum_{i, j=1}^{N} \tilde{w}_{i j}(u) u \partial_{i j}^{2} u,
\end{aligned}
$$

with $w_{0}(u), w_{i}(u), w_{i j}(u)$ and $\tilde{w}_{i j}(u)$ all in $L^{\infty}$. Now, $u=e^{-s \rho} v_{0}$ for some $s>0$ and $v_{0} \in W^{2, p}$ by Theorem 6.3. By substitution into (6.8), we find that $L(0) u-$ $F(u)=e^{-2 s \rho} g_{0}$ with $g_{0} \in L^{p}$ depending upon $v_{0}$. Note that $g_{0} \in L^{p}$, since $v_{0}^{2}, v_{0} \partial_{i} v_{0}, \partial_{i} v_{0} \partial_{j} v_{0}$ and $v_{0} \partial_{i j} v_{0}$ are all in $L^{p}$. Since $F(u)=f$, this means that $u$ solves the equation

$$
L(0) u=f+e^{-2 s \rho} g_{0} .
$$

Since $e^{t \rho} f \in L^{p}$, then also $e^{\min (t, 2 s) \rho}\left(f+e^{-2 s \rho} g_{0}\right) \in L^{p}$. By Theorem 3.4 for $L(0)$, we find $u=e^{-\min \left(s_{0}, \min (t, 2 s)\right) \rho} v_{1}$ with $v_{1} \in W^{2, p}$. If $t \leq 2 s$, this is the desired result that $u=e^{-\min \left(s_{0}, t\right) \rho} v_{1}$ with $v_{1} \in W^{2, p}$. If $t>2 s$, then $u=e^{-\min \left(s_{0}, 2 s\right) \rho} v_{1}$, which is the same relation as $u=e^{-s \rho} v_{0}$ above with $s$ replaced by $\min \left(s_{0}, 2 s\right)$. Thus, by the same procedure (i.e., let $u=e^{-\min \left(s_{0}, 2 s\right) \rho} v_{1}$ in the right-hand side of (6.8)), we arrive at $u=e^{-\min \left(s_{0}, \min \left(t, 2 \min \left(s_{0}, 2 s\right)\right)\right) \rho} v_{2}$ with $v_{2} \in W^{2, p}$. But $\min \left(s_{0}, \min \left(t, 2 \min \left(s_{0}, 2 s\right)\right)\right)=\min \left(s_{0}, \min (t, 4 s)\right)$, so that $u=e^{-\min \left(s_{0}, \min (t, 4 s)\right) \rho} v_{2}$.

If $t \leq 4 s$, this is $u=e^{-\min \left(s_{0}, t\right) \rho} v_{2}$, as desired. If $t>4 s$, then $u=e^{-\min \left(s_{0}, 4 s\right) \rho} v_{2}$ and we are in the same situation as before with $2 s$ replaced by $4 s$. By repeating the operation, we get $u=e^{-\min \left(s_{0}, t\right) \rho} v_{k}$ if $t \leq 2^{k} s$ or $u=e^{-\min \left(s_{0}, 2^{k} s\right) \rho} v_{k}$ if $t>2^{k} s$, for some $v_{k} \in W^{2, p}$ and every $k \in \mathbb{N}$. Evidently, $t \leq 2^{k} s$ occurs for $k$ large enough and then $u=e^{-\min \left(s_{0}, t\right) \rho} v_{k}$. This completes the proof.

A result in the spirit of Corollary 6.4 is given in [27, Theorem 2.1] for the case when $\rho(x)=|x|$ for $|x|$ large enough (exponential decay). No Fredholmness assumption is made in [27], and indeed degenerate ellipticity is allowed, but the coefficients must be real and $\underline{\lim }_{|x| \rightarrow \infty} c(x, 0)>0$ is needed. Thus, in the linear case, that theorem can only be applied to real eigenvalues $\lambda$ satisfying $\lambda<\underline{\lim }_{|x| \rightarrow \infty} c(x)$. The method of proof in 27], based on the maximum principle, does not lend itself to generalizations to higher order problems, systems or complex coefficients.

As a special case of Theorem 6.3 and Corollary 6.4 we obtain the exponential decay of the solutions of $F(u)=0$ :

Corollary 6.5. Let $\rho \geq 0$ be a $C^{2}$ function such that $\rho(x)=|x|$ for $|x|$ large enough. Let $p \in(N, \infty)$ be given and suppose that the nonlinear operator $F$ in (6.1) is Fredholm from $W^{2, p}$ to $L^{p}$. Then, every solution $u \in W^{2, p}$ of $F(u)=0$ has the form $u=e^{-s \rho} v$ for some $s>0$ independent of $\rho$ and some $v \in W^{2, p}$. Furthermore, if the coefficients $b_{i}$ and $c$ are $C_{\xi}^{1}$ bundle maps such that $\partial_{\xi_{j}} b_{i}(\cdot, 0)$ $\in L^{\infty}$ and $\partial_{\xi_{j}} c(\cdot, 0) \in L^{\infty}$ for $0 \leq j \leq N$, then $s$ depends only upon $D F(0)$.

Remark 4.1 can be repeated: In Corollary 6.5, $u \in \bigcap_{1 \leq r \leq p} W^{2, r}$. If also $\nu=0$, all the coefficients are real and $\gamma$ is independent of $x$ in (6.4), then $u \in \bigcap_{1 \leq r<\infty} W^{2, r}$.

The existence of nontrivial solutions of $F(u)=0$ is known for some $F$, sometimes, but not always, along with their exponential decay. See Berestycki and Lions [7], Chaljub and Volkman [10], Coti Zelati and Rabinowitz [11], Kryszewski and 
Szulkin [23], Lions [25], Rother [31, Strauss [33], Troestler and Willem [34. Theorem 6.3 and Corollary 6.5 show that the exponential decay property is completely general. As a rule, existence is only proved in $W^{1,2}$, but membership in $W^{2, p}$ can be established by bootstrapping arguments.

In a different direction, the exponential decay of the solutions of $F(u)=0$ can also be used to prove the nonexistence of nontrivial solutions via Pohozaev identities; see [27.

Remark 6.3. If the coefficients $a_{i j}$ do not depend upon $\xi_{0}$ and $b$ does not depend upon $\left(\xi_{1}, \ldots, \xi_{N}\right)$, then Theorem 6.3 and Corollary 6.5 remain valid for $p \in$ $(\max (1, N / 2), \infty)$ and $a_{i j} \in W_{l o c}^{1, \infty}$ instead of (6.2) and (6.3). See Remark 6.2 (iii).

\section{IsOlATED EIGENVALUES OF FINITE MULTIPLICITY}

In this section, we give an alternate and completely elementary proof of Theorem 4.1 when $\lambda$ is an isolated eigenvalue of $L$ of finite multiplicity (in particular, $\nu=0$ ) under slightly different assumptions: We assume that the coefficients $a_{i j}$ are real, bounded and uniformly continuous and that $b_{i}, c \in L^{\infty}$, possibly complex-valued. We also assume that the uniform ellipticity condition

$$
\sum_{i, j=1}^{N} a_{i j}(x) \eta_{i} \eta_{j} \geq \gamma|\eta|^{2}, \quad \forall \eta=\left(\eta_{1}, \ldots, \eta_{N}\right) \in \mathbb{R}^{N},
$$

holds, where $\gamma>0$ is a constant independent of $x, \eta$.

In that case, it is well-known that the operator $L$ is a closed unbounded operator on $L^{p}$ with domain $W^{2, p}$ for every $p \in(1, \infty)$; see Browder 8 . Recall that the closedness of $L$ follows from the a priori estimate

$$
\|\left. u\right|_{2, p} \leq C\left(|u|_{0, p}+|L u|_{0, p}\right),
$$

for $u \in W^{2, p}$, where $C>0$ is a constant independent of $u$. In turn, (7.2) can be derived from the classical local a priori estimates and, for the specific case of $\mathbb{R}^{N}$ of interest here, a simple cube decomposition of the space ([26]). Of course, $L \in \mathcal{L}\left(W^{2, p}, L^{p}\right)$ as well, and (7.2) expresses the equivalence of the $W^{2, p}$ norm and the graph norm on $D(L)=W^{2, p}$.

In what follows, $L_{s}$ continues to denote the operator (1.2). Although Lemma 7.1 is valid for a general $\rho$, it will be sufficient to consider the case when the $C^{2}$ function $\rho \geq 0$ coincides with $|x|$ for $|x|$ large enough. It is obvious from (2.3) that the coefficients of $L_{s}$ satisfy the same conditions as the coefficients of $L$. Hence, $L_{s} \in \mathcal{L}\left(W^{2, p}, L^{p}\right)$ and $L_{s}$ is a closed unbounded operator on $L^{p}$ with domain $W^{2, p}$.

Lemma 7.1. Let $p \in(1, \infty)$ be given and let $L$ and $L_{s}$ be viewed as closed unbounded operators on $L^{p}$ with domain $W^{2, p}$. Suppose that 0 is an eigenvalue of $L$ of finite multiplicity $m$. Then, there is $s>0$ such that 0 is an isolated eigenvalue of $L_{s}$ of multiplicity $m$.

Proof. First, the gap (in the sense of Kato [22]) $\hat{\delta}\left(L_{s}, L\right)$ tends to 0 with $s$. To see this, argue by contradiction, assuming there is a sequence $\left(s_{n}\right)$ tending to 0 such that $\hat{\delta}\left(L_{s_{n}}, L\right)$ is bounded away from 0 . A contradiction then arises from 22 . Theorem 2.29, p. 207] with (using the notation of that theorem) $X=Y=L^{p}$,

\footnotetext{
${ }^{8}$ Another argument is that the gap between the graphs of $L$ and $L_{s}$ is equivalent to the norm of $L-L_{s}$ in $\mathcal{L}\left(W^{2, p}, L^{p}\right)$, but there is no convenient reference in [22].
} 
$Z=W^{2, p}, T=L, T_{n}=L_{s_{n}}$ and with $U_{n}=U$ being the embedding $W^{2, p} \hookrightarrow L^{p}$ : Lemma 2.1] with $s=s_{n}$ is the condition $\left\|V_{n}-V\right\| \rightarrow 0$ of [22, Theorem 2.29, p. 207], which in turn implies that $\hat{\delta}\left(L_{s_{n}}, L\right)$ tends to 0 and provides the desired contradiction.

Now, it follows from [22, p. 213] that when $|s|>0$ is small enough, the spectrum of $L_{s}$ near 0 consists of finitely many isolated eigenvalues $\lambda_{s, 1}, \ldots, \lambda_{s, k(s)}$ with multiplicities $m_{s, 1}, \ldots, m_{s, k(s)}$, respectively, satisfying $m_{s, 1}+\ldots+m_{s, k(s)}=m$. More precisely, $\lambda_{s, 1}, \ldots, \lambda_{s, k(s)}$ lie on a disk $D \subset \mathbb{C}$ centered at 0 , in which $L$ has no eigenvalue other than 0 . We claim that if also $s>0$, then $k(s)=1$ and $\lambda_{s, 1}=0$ (whence $m_{s, 1}=m$, and the proof is complete). Otherwise, $L_{s}$ has an eigenvalue $\lambda_{s} \in D$ with $\lambda_{s} \neq 0$. If $v \in W^{2, p} \backslash\{0\}$ is a corresponding eigenfunction, then $u:=e^{-s \rho} v \in$ $W^{2, p} \backslash\{0\}$ since $s>0$, and $L u-\lambda_{s} u=L\left(e^{-s \rho} v\right)-\lambda_{s} e^{-s \rho} v=e^{-s \rho}\left(L_{s}-\lambda_{s}\right) v=0$. Thus, $\lambda_{s} \in D$ is an eigenvalue of $L$ distinct from 0 , which is absurd.

The exponential decay of the generalized eigenfunctions is embodied in

Theorem 7.2. Let $\rho \geq 0$ be a $C^{2}$ function such that $\rho(x)=|x|$ for $|x|$ large enough. Let $p \in(1, \infty)$ be given and let $L$ be viewed as a closed unbounded operator on $L^{p}$ with domain $W^{2, p}$. If $\lambda$ is an isolated eigenvalue of finite multiplicity, there is $s_{\lambda}>0$, independent of $\rho$, such that every generalized eigenfunction $u$ of $L$ associated with $\lambda$ has the form $u=e^{-s_{\lambda} \rho} v$ for some $v \in W^{2, p}$.

Proof. After replacing $L$ by $L-\lambda$ we may assume that $\lambda=0$. The generalized null-space of $L$ is defined as the range of the projection $\frac{1}{2 \pi i} \int_{\Gamma}(L-\mu)^{-1} d \mu$, where $\Gamma$ is a small circle around 0 . As in the finite dimensional case, it also coincides with $\operatorname{ker} L^{k_{0}}$ for the smallest integer $k_{0}$ such that the sequence ker $L^{k}$ stabilizes (see for instance [6. Theorem 7.3, p. 257]), and hence $m:=\operatorname{dim} \operatorname{ker} L^{k_{0}}$ is (by definition) the multiplicity of the eigenvalue 0 . With $s>0$ given by Lemma 7.1 a similar result holds with $L_{s}$ instead of $L$, namely $m=\operatorname{dim} \operatorname{ker} L_{s}^{k_{s}}$ for some integer $k_{s}$.

Now let $v \in \operatorname{ker} L_{s}^{k_{s}}$, so that $L_{s}^{j} v \in W^{2, p}$ for $0 \leq j \leq k_{s}-1$ and $L_{s}^{k_{s}} v=0$. Set $u:=e^{-s \rho} v \in W^{2, p}$. Since $L u=e^{-s \rho} L_{s} v$, we infer that $L u \in W^{2, p}$. More generally, $L^{j} u=e^{-s \rho} L_{s}^{j} v \in W^{2, p}$ for $0 \leq j \leq k_{s}-1$. In particular, $L^{k_{s}} u=e^{-s \rho} L_{s}^{k_{s}} v=0$, i.e., $u \in \operatorname{ker} L^{k_{s}} \subset \operatorname{ker} L^{k_{0}}$ (since $\operatorname{ker} L^{k} \subset \operatorname{ker} L^{k_{0}}$ for all $k$ ).

Above, let $v=v_{i}$, where $\left\{v_{1}, \ldots, v_{m}\right\}$ is a basis of $\operatorname{ker} L_{s}^{k_{s}}$, so that $\left\{u_{1}, \ldots, u_{m}\right\}$ with $u_{i}:=e^{-s \rho} v_{i}$ are in $\operatorname{ker} L^{k_{0}}$. It is trivial that $\left\{u_{1}, \ldots, u_{m}\right\}$ is linearly independent and hence a basis of ker $L^{k_{0}}$. Therefore, every generalized null-function of $L$ is a linear combination of $\left\{u_{1}, \ldots, u_{m}\right\}$ and hence has the form $u=e^{-s \rho} v$ for some $v \in W^{2, p}$.

In a less precise form and if all the coefficients are uniformly continuous, Theorem 7.2 was recently proved by Angenent [4 via estimates for the resolvent obtained from properties of holomorphic semigroups. When $L$ is a Schrödinger operator, a proof of Theorem 7.2 is given in [32, Theorem C.3.4], based on an argument of Aguilar and Combes [2] closely related to Lemma 7.1, but relying on a more complicated analytic continuation argument. The method of proof of Theorem 7.2 can readily be used with general elliptic systems of arbitrary order (also discussed in [4]), in various functional settings. 


\section{REFERENCES}

[1] Agmon, S., Lectures on exponential decay of solutions of second-order elliptic equations: bounds on eigenfunctions of $N$-body Schrödinger operators, Mathematical Notes, Vol. 29, Princeton University Press, Princeton (1982). MR 85f:35019

[2] Aguilar, J. and Combes, J. M., A class of analytic perturbations for one-body Schrödinger Hamiltonians, Comm. Math. Phys. 22 (1971), 269-279. MR 49:10287

[3] Alinhac, S. and Baouendi, M. S., Uniqueness for the characteristic Cauchy problem and strong unique continuation for higher order partial differential inequalities, Amer. J. Math. 102 (1980), 179-217. MR 81e:35003

[4] Angenent, S., Constructions with analytic semigroups and abstract exponential decay results for eigenfunctions, Progress in Nonlinear Differential Equations and Their Applications, Vol. 35, Birkhäuser, Basel (1999), 11-27. MR 2000h:47006

[5] Anane, A., Chakrone, O., El Allali, Z. and Hadi, I., A unique continuation property for linear elliptic systems and nonresonance problems, Electron. J. Differential Equations 46 (2001) (electronic). MR 2002j:35090

[6] Bellini-Morante, A., Applied Semigroups and Evolution Equations, Oxford Mathematical Monographs, Oxford (1979). MR 82f:47001

[7] Berestycki, H. and Lions, P.-L., Nonlinear scalar field equations, Arch. Rat. Mech. Anal. 82 (1983), 313-376. MR 84h:35054a

[8] Browder, F. E., On the spectral theory of elliptic differential operators I, Math. Ann. 142 (1960/61), 22-130. MR 35:804

[9] Caffarelli, L. A. and Friedman, A., Partial regularity of the zero-set of solutions of linear and superlinear elliptic equations. J. Differ. Eqns. 60 (1985), 420-433. MR 87e:35006

[10] Chaljub, A. and Volkmann, P., Existence of ground states and exponential decay for semilinear elliptic equations in $\mathbb{R}^{N}$, J. Differ. Eqns. 76 (1988), 374-390. MR 90c:35076

[11] Coti Zelati, V. and Rabinowitz, P. H., Homoclinic type solutions for a semilinear elliptic PDE on $\mathbb{R}^{N}$, Comm. Pure Appl. Math. 45 (1992), 1217-1269. MR 93k:35087

[12] Dautray, R. and Lions, J.-L., Mathematical Analysis and Numerical Methods for Science and Technology, Springer-Verlag, Berlin (2000).

[13] De Carli, L., Unique continuation for a class of higher order elliptic operators, Pacific J. Math. 179 (1997), 1-10. MR 98d:35046

[14] De Carli, L., Unique continuation for elliptic operators with non-multiple characteristics, Israel J. Math. 118 (2000), 15-27. MR 2001f:35074

[15] Dehman, B. and Robbiano, L., La propriété du prolongement unique pour un système elliptique: Le système de Lamé, J. Math. Pures Appl. 72 (1993), 475-492. MR 94h:35051

[16] de Figueiredo, D. G. and Gossez, J.-P., Strict monotonicity of eigenvalues and unique continuation, Comm. Partial Differ. Eqns. 17 (1992), 339-346. MR 93b:35098

[17] Garofalo, N. and Lin, F. H., Unique continuation for elliptic operators: a geometricvariational approach, Comm. Pure Appl. Math. 40 (1987), 347-366. MR 88j:35046

[18] Glazman, I. M., Direct Methods of Qualitative Spectral Analysis of Singular Differential Operators, Israel Prog. Sci. Trans., Jerusalem (1966). MR 32:8210

[19] Gossez, J.-P. and Loulit, A., A note on two notions of unique continuation, Bull. Soc. Math. Belg., Ser. B 45 (1993), 257-268. MR 96k:35034

[20] Hislop, P. D., Exponential decay of two-body eigenfunctions: a review, Proceedings of the Symposium on Mathematical Physics and Quantum Field Theory (Berkeley, CA, 1999), 265-288 (electronic), Electron. J. Differ. Equ. Conf., 4, Southwest Texas State Univ., San Marcos, TX, 2000. MR 2001j:81247

[21] Hislop, P. D. and Sigal, I. M., Introduction to Spectral Theory, Springer-Verlag, Berlin (1996). MR 98h:47003

[22] Kato, T., Perturbation theory for linear operators. Springer-Verlag, New York, (1980). MR 96a:47025 (reprint)

[23] Kryszewski, W. and Szulkin, A., Generalized linking theorem with an application to a semilinear Schrödinger equation, Adv. Diff. Eqns. 3 (1998), 441-472. MR 2001g:58021

[24] Lindenstrauss, J. and Tzafriri, L., Classical Banach spaces I and II, Springer-Verlag, Berlin (1996). MR 58:17766, MR 81c:46001 (1st eds.)

[25] Lions, P.-L., Solutions of the Hartree-Fock equations for Coulomb systems, Comm. Math. Phys. 109 (1987), 33-97. MR 88e:35170 
[26] Rabier, P. J., Invariance of the $\Phi_{0}$-spectrum and Sobolev regularity for second order linear elliptic problems on $\mathbb{R}^{N}$ in "Applicable Mathematics in the Golden Age", J. C. Misra Ed., Narosa Publishing House, New Dehli (2003), 1-31.

[27] Rabier, P. J. and Stuart, C. A., Exponential decay of the solutions of quasilinear second-order equations and Pohozaev identities, J. Diff. Eqns. 165 (2000), 199-234. MR 2001f:35139

[28] Rabier, P. J. and Stuart, C. A., Fredholm properties of Schrödinger operators in $L^{p}\left(\mathbb{R}^{N}\right)$, Diff. Int. Eqns. 13 (2000), 1429-1444. MR 2001m:47103

[29] Rabier, P. J. and Stuart, C. A., Fredholm and properness properties of quasilinear elliptic operators on $\mathbb{R}^{N}$, Math. Nachr. 231 (2001), 129-168. MR 2003c:35026

[30] Robbiano, L., Dimension des zéros d'une solution faible d'un opérateur elliptique, J. Math. Pures Appl. 67 (1988), 339-357. MR 90c:35059]

[31] Rother, W., Nonlinear scalar field equations, Differ. Integral Eqns. 5 (1992), 777-792. MR 93e:35030

[32] Simon, B., Schrödinger semigroups, Bull. Amer. Math. Soc. 7 (1982), 447-526. MR 86b:81001a

[33] Strauss, W.A., Existence of solitary waves in higher dimension, Comm. Math. Phys. 55 (1977), 149-162. MR 56:12616

[34] Troestler, C. and Willem, M., Nontrivial solution of a semilinear Schrödinger equation, Comm. PDEs 21 (1996), 1431-1449. MR 98i:35034

[35] Wang, W., Carleman inequalities and unique continuation for higher-order elliptic differential operators, Duke Math. J. 74 (1994), 107-128. MR 95j:35078

Department of Mathematics, University of Pittsburgh, Pittsburgh, Pennsylvania 15260

E-mail address: rabier@imap.pitt.edu 